\title{
Enmascaramiento vs. Oclusión en la evaluación para el implante BAHA con hipoacusia neurosensorial profunda unilateral
}

\author{
Gloria Andrea Ocampo Lozano* \\ Claudia Marcela Triana Gómez**
}

\section{Resumen}

Con el objetivo de determinar los umbrales auditivos y contrastarlos con los procedimientos de enmascaramiento mínimo y máximo con los de oclusión en usuarios con hipoacusia neurosensorial profunda unilateral, candidatos a implante BAHA, se tomaron 16 candidatos de la Clínica José A. Rivas, los cuales primero fueron evaluados otológicamente y se les descartó alguna patología retrococlear. Ellos decidieron voluntariamente su participación en la investigación y firmaron el consentimiento informado. Se les realizó otoscopia y membrana timpánica, luego la audiometría que evaluó los umbrales mínimos de audición, adicionalmente se realizó la logoaudiometría y la inmitancia acústica para corroborar la función del oído medio. Seguidamente se realizó la evaluación de audiometría tonal con el BAHA utilizando en el oído con la hipoacusia el procesador DIVINO en campo libre con el enmascaramiento en donde se aplicó un ruido de $15 \mathrm{~dB}$ por encima del umbral en el oído con audición normal y 30 dB por encima del umbral aéreo para el masking máximo. Luego se realizó la oclusión, introduciendo material de impresión cubriendo totalmente el CAE. Para los dos procedimientos se tomaron los umbrales de las frecuencias $500 \mathrm{~Hz}, 1000 \mathrm{~Hz}, 2000 \mathrm{~Hz}$ y $4000 \mathrm{~Hz}$ con el parlante a 0 grados azimut. Se encontraron diferencias importantes entre los dos procedimientos; siendo la oclusión, el mejor procedimiento para la evaluación de implante BAHA.

Palabras clave: enmascaramiento, oclusión, audiometría tonal.

\footnotetext{
Fonoaudióloga. Especialista en Audiología. Clínica Rivas. glohen1981@ hotmail.com

** Fonoaudióloga. Especialista en Audiología. Clínica Rivas. Consultorio particular
} 


\section{Masking $V_{s}$ Occlusion in the assessment for the BAHA implant with deep unilateral sensorineural hypoacusism}

\section{Abstract}

In order to determine the hearing thresholds and compare them with masking procedures with the minimum and maximum occlusion in users with deep unilateral sensorineural Hypoacusis, 16 BAHA candidates were taken from The Jose A. Rivas Clinic, who were first evaluated otológically and as a result they got negative any retrocochlear pathology. They decided voluntarily their participation in the research and signed informed consent. It was performed an otoscopy and tympanic membrane, then an audiometry that evaluated their minimal hearing thresholds, in addition it was made a logoaudiometry and an acoustic immittance to corroborate the function of the middle ear. Then an evaluation was developed by doing a tonal audiometry using the BAHA in the ear with hypoacusis using the "DIVI$N O$ " processor in an open place with the masking where it was applied a noise of $15 \mathrm{~dB}$ above the threshold in the ear with normal hearing and $30 \mathrm{~dB}$ above the air threshold for the maximum masking. Then the occlusion was performed by introducing impression material covering the entire CAE. For both procedures the thresholds for the frequencies were $500 \mathrm{~Hz}, 1000 \mathrm{~Hz}, 2000 \mathrm{~Hz}$ and $4000 \mathrm{~Hz}$ with the speaker at 0 degrees azimuth. We found significant differences between the procedures, the occlusion is the best procedure for the evaluation of a BAHA implant.

Key word: masking, occlusion, Tonal audiometry.

La audiología se encarga del estudio de la audición, lo que permite analizarla desde la normalidad y el desorden, el audiólogo es un profesional quien por virtud de su entrenamiento académico clínico, está calificado para proveer el servicio profesional relacionado con la prevención, evaluación y rehabilitación de los impedimentos auditivos ${ }^{1}$; por lo que se encarga del estudio de los estímulos vibrantes captados por el oído, los que finalmente van a ser interpretados por la corteza cerebral, de esta forma el oído es una de las modalidades sensoriales de mayor importancia para relacionarnos adecuadamente con el entorno.

Asoaudio, audiología en general. [online]. www.asoaudio.org.co
Es por esto que las personas con pérdida auditiva tienen graves problemas en la comunicación. Su nivel de problemática depende de varios factores; entre los cuales se destaca prioritariamente el grado de pérdida auditiva, el nivel y naturaleza de discriminación de la percepción del habla, edad y forma de aparición, estrategias de compensación usadas por el individuo para suplir la pérdida auditiva y las necesidades comunicativas del sujeto ${ }^{2}$.

Dentro de la audiología, área incluida dentro de la fonoaudiología, la medición es uno de los aspectos de mayor importancia, porque permite la asignación de cifras en forma específica, y analiza el nivel de audición, lo que determina la normali-

\footnotetext{
Ibíd., pág. 214.
} 
dad en este proceso o las diferentes alteraciones auditivas. La evaluación de la audición, cumple con numerosas funciones: Guía a la habilitación, a la rehabilitación y al control, así como también permite evaluar la efectividad de la intervención.

Se requiere por lo tanto, una especificación minuciosa de dicho procedimiento, para que se haga una meticulosa cuantificación de las mediciones y la observación de las respuestas de los estímulos, lo que le dará mayor confiabilidad a determinada prueba.

La hipoacusia es caracterizada por una reducción de la sensibilidad del mecanismo auditivo, por lo que el individuo requiere que la intensidad de los sonidos sea aumentada para poder percibirlos. Generalmente es causada por un daño de las células sensoriales, neurales, o por un mal funcionamiento de las conexiones dentro de la cóclea ${ }^{3}$, entonces la hipoacusia se refiere a la disminución de la audición ${ }^{4}$. O a un trastorno auditivo en aquel oído que tiene una desviación significativa con respecto al comportamiento del oído normal promedio ${ }^{5}$.

Dependiendo del sitio de lesión del oído, la hipoacusia se clasifica en conductiva, en la cual se ve afectada la transmisión del sonido por el oído externo y el medio por factores infecciosos, obstructivos e inflamatorios y se caracteriza por tener descenso en vía aérea con conservación ósea; la hipoacusia mixta que es la pérdida auditiva en la cual se encuentran afectados tanto el oído medio como el interno, suele estar asociada con traumatismos severos y múltiples, clínicamente existe alteración en el mecanismo de la conducción como en el de la percepción: La vía aérea como la ósea están descendidas en el mismo oído,

Gómez Gómez, Olga. Audiología básica. Bogotá Colombia: Universidad Nacional de Colombia, 2006, pág. 72.

Carcedo Gracia, Luis Maria. Otología. Op. cit., pág. 80.

Rivas, José A. Tratado de Otología y Audiología. Op. cit., pág. 94. pero entre ellas se puede encontrar una diferencia de más de $10 \mathrm{~dB}$; por último, la hipoacusia neurosensorial también llamada sensoneural que ocurre cuando el sonido es conducido adecuadamente por el canal auditivo externo (CAE) y el oído medio hasta los líquidos del oído interno pero, éste no puede ser percibido y analizado normalmente ya que los mecanismos conductivos de la cóclea se encuentran comprometidos.

Dependiendo del oído comprometido, se divide en hipoacusia unilateral que es el término utilizado para definir la pérdida auditiva de un solo oído con conservación del otro, puede estar presente al nacer o desarrollarse después. La sordera unilateral se debe a infecciones virales como otitis a repetición, la viruela, la meningitis; también se produce por trauma craneoencefálico, el neurinoma acústico, tumores en oído medio e interno, pérdida auditiva súbita y cirugías en el oído ${ }^{6}$.

Este tipo de pérdida, también puede llamarse hipoacusia neurosensorial unilateral, causada por varios factores como fracturas translaberínticas, extirpación de neurinoma del acústico, sordera súbita, entre otras ${ }^{7}$.

En las personas que tienen este tipo de pérdida, no se hacen tan evidentes las problemáticas sociales, laborales y/o escolares, dado que el oído contrario suple y equipara la función auditiva binaural; sin embargo, ellos refieren presentar dificultades para desenvolverse socialmente, en el trabajo y en los diferentes ámbitos donde la persona se desenvuelve.

La población con pérdida unilateral presenta dificultad para comprender los sonidos del lenguaje en ambientes ruidosos; en la localización de sonidos ya que se les dificulta determinar la procedencia y ubicación de ellos, especialmente si hay ruido

\footnotetext{
Gómez Gómez, Olga. Audiología básica. Op. cit., págs. 72-73.

Carcedo Gracia, Luis María. Otología. Buenos Aires, Madrid: Panamericana, 2004, págs. 280-292-319.
} 
de fondo, todo esto limita seriamente las habilidades para desenvolverse adecuadamente en actividades sociales. Además, esta población debe realizar compensación con el oído sano para superar los obstáculos para la discriminación, pueden presentar tinnitus, problemas en el equilibrio y muchos terminan aislándose socialmente. Los pacientes con pérdida auditiva unilateral, también pueden presentar irritabilidad, dolores de cabeza frecuentes por tensión, aislamiento social, vértigos, inhabilidad para aislar el ruido de fondo o aislar selectivamente en ruido y comportamiento evasivo al no entender el discurso ${ }^{8}$.

La hipoacusia bilateral, es la pérdida de audición permanente en ambos oídos, que se origina en disfunción conductiva y/o sensorial de algún componente del sistema auditivo, con el consecuente deterioro de la comunicación en estas personas.

En los últimos años se ha avanzado en la investigación tecnológica y en la forma de adaptar con efectividad las prótesis auditivas, generalizándose en una forma asombrosa el uso de éstas. Hoy en día se encuentra gran cantidad de temores con respecto al uso, por razones estéticas, desinformación y por falsas creencias, por lo que es de gran importancia, que la adaptación de las prótesis auditivas sea hecha por un profesional competente en este tipo de situaciones.

Pero hay que tener en cuenta que la pérdida auditiva por sí misma, no determina que una persona sea candidata para la amplificación, la necesidad de comunicación del individuo es el factor primario y determinante, la preselección de las ayudas auditivas está determinada por las pruebas audiométricas realizadas sin audífonos entre las cuales se encuentran: la impedanciometría, los umbrales auditivos vía aérea y vía ósea, los niveles

Arlinger, S. Negative consequences of uncorrected hearing loss - a review. University Hospital, Department of Audiology [online], july 2003. Pub med - indexed for MEDLINE. de discriminación y el nivel de comodidad e incomodidad ante dichas ayudas auditivas.

La amplificación de las pérdidas auditivas tienen ciertas especificaciones para ser adaptados, las ganancias en habilidades auditivas, comunicativas y los beneficios psicológicos, sociales y educativos que se obtienen, responden no sólo a las características individuales de la persona, sino también a las condiciones técnicas y humanas que ofrezca cada programa de implante en particular ${ }^{1}$.

Existen diferentes tipos de prótesis auditivas dependiendo del déficit auditivo y la buena solución que ésta dé a las necesidades; por esta razón es importante someter a la persona afectada a una selección apropiada de prótesis auditiva, ya sea para la pérdida auditiva unilateral o bilateral.

El proceso de selección se inicia con la consideración del tipo de audífono que se va a utilizar teniendo en cuenta los resultados de la audiometría tonal $^{9}$.

Actualmente existen diferentes tipos de ayudas auditivas; entre éstos están las ayudas convencionales y las no convencionales. Dentro de las primeras, están los llamados audífonos o prótesis auditivas de estimulación auditiva, las que incluyen un amplificador de sonido, construido de tal forma que pueda llevarse lo más cómodamente posible, aumentando la potencia sonora y dando al paciente con pérdida auditiva la intensidad que necesita. Pero éstos tienen como restricción su uso en pérdidas auditivas profundas porque no logra la suficiente estimulación e intensidad para amplificar este grado de pérdidas auditivas.

Los no convencionales, en los que se encuentran el Sistema Crossover que funciona a través de conducción ósea en donde el sonido se transfiere a través del hueso del cráneo por medio de un audífono muy pequeño que estimula la cóclea del oído por el

Rivas, Tratado de Otología y Audiología. Op. cit., pág. 620. 
que escucha, el cerebro es capaz de distinguir entre el sonido que recibe del oído hipoacúsico vía audífono, del sonido que recibe directamente del oído que puede escuchar normalmente; el resultado final es que el paciente tiene la sensación de escuchar a través del oído hipoacúsico ${ }^{10}$.

Para las personas con hipoacusia neurosensorial unilateral a quienes no se les puede adaptar los audífonos convencionales, se pensó en implementar sistemas de vibración directa al cráneo para aprovechar la transmisión ósea del sonido de los huesos del cráneo, entre estos sistemas se encuentran:

El Sistema Cros que beneficia a personas con problemas auditivos unilaterales ya que consiste en hacer rotar la señal acústica de un lado al otro lado para ser mejor percibida, se adapta en casos con sensibilidad auditiva periférica normal en un oído y una pérdida auditiva severa o profunda del otro oído, se coloca un micrófono en el lado sordo, el cual envía la señal al oído bueno. Sin embargo, los éxitos recientes con los audífonos osteointegrados BAHA en las sorderas profundas unilaterales han reducido su aplicabilidad ${ }^{11}$.
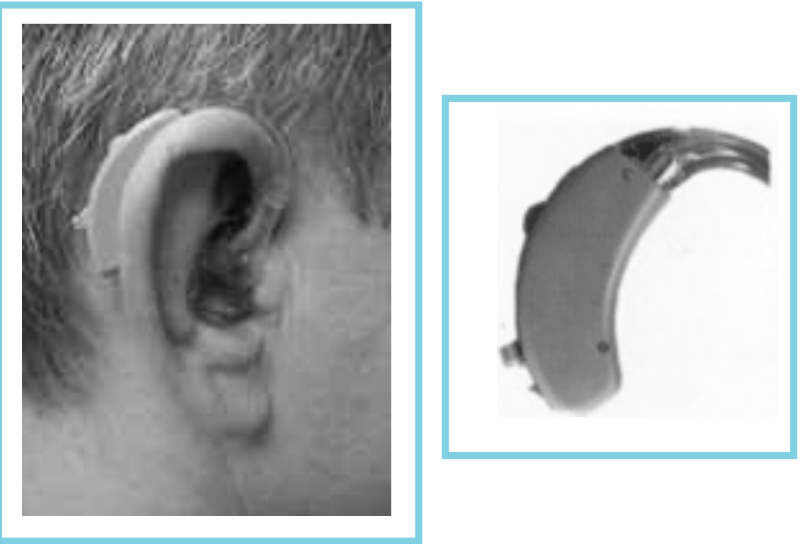

Figura 1. Audífono Cros Retroauricular.

10 Cómo funciona el BAHA. www.spain.cochlear.com/Products/ 1755.asp - 8k

11 Rivas, José A. Otología. Op. cit., pág. 618.
Las prótesis auditivas osteointegradas, conocidas como BAHA (Bone Anchored Hearing Aid), utilizan la vía ósea en lugar de la aérea para transmitir el sonido hasta el oído interno, como se aprecia en la figura 2.
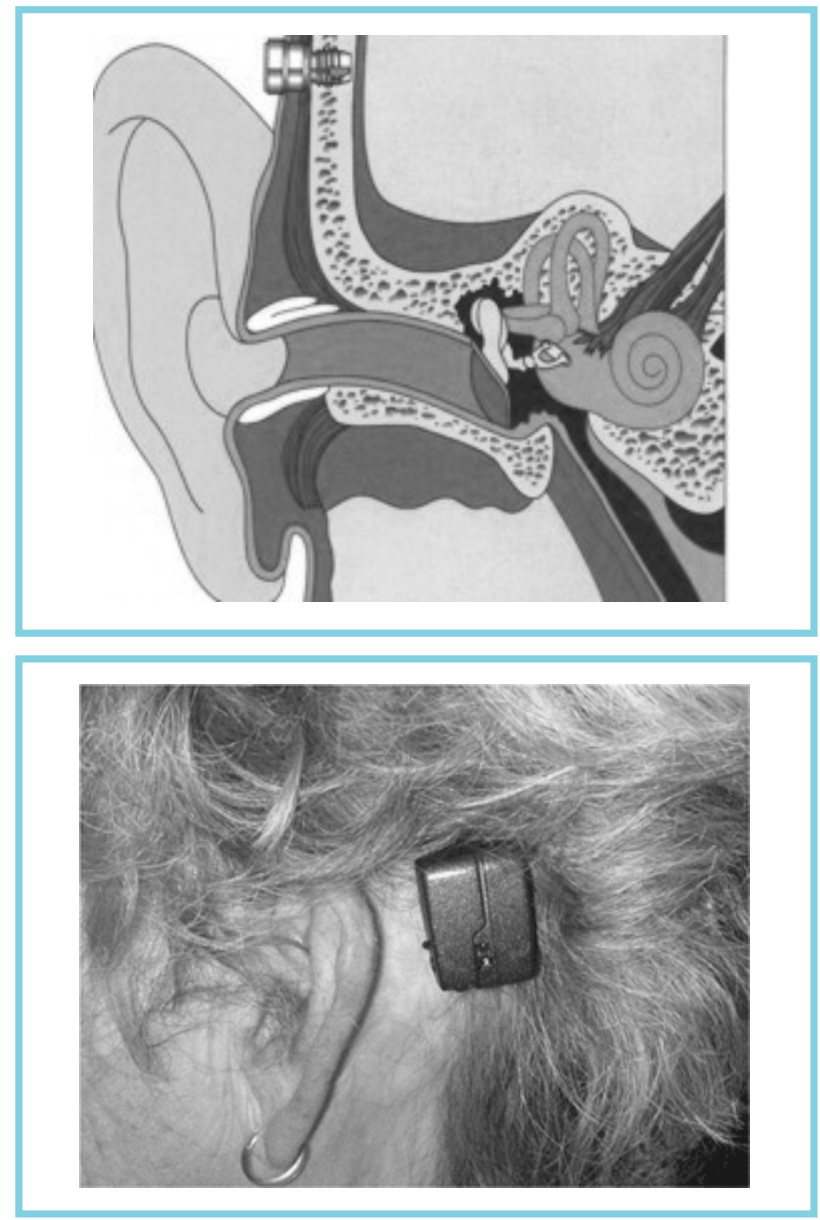

Figura 2. BAHA.

La intervención necesaria para su colocación es sencilla y consiste en implantar un tornillo de titanio con el fin de que se integre o haya una íntima adhesión de este material al hueso.

Existen dos opciones a la hora de plantear la estrategia quirúrgica: a) colocar el tornillo y esperar varios meses a su integración, en una segunda intervención colocar el pedestal externo y b) realizarlo al mismo tiempo arriesgando una posible infección por la puerta de entrada al perforar la piel. El colgajo de piel que rodea el pedestal debe estar libre de folículos pilosos y quedar muy delgado 
para permitir la colocación posterior del componente externo.

Se pueden beneficiar los pacientes que presentan hipoacusia conductiva bilateral o mixta, por infecciones de oído, atresias de Conducto Auditivo Externo y malformaciones de oído medio que imposibilitan el uso de audífonos y en las que la cirugía no está indicada, algunas veces en personas con pérdida auditiva neurosensorial unilateral.

El BAHA: consta de un audífono externo que hace que la audición se transmita por vía ósea hasta el oído interno, obviando el oído medio y externo; sin embargo, la función del nervio auditivo debe estar bien conservada, además tiene un poste de titanio implantado en el hueso por medio de una intervención quirúrgica, audífono vibrador fijado al tornillo de titanio y una batería.

Actualmente se pueden conseguir tres componentes del BAHA:

El BAHA Divino: provee sonido digital y sistema de micrófono direccional, óptima señal en rui- do, sistema de ajuste AGCo, 2 programas con audición omnidireccional y direccional para conversación directa, batería de 13. Potencia en pérdidas auditivas hasta $40 \mathrm{~dB}$ y pérdidas neurosensoriales profundas.

El BAHA Intenso: es el más reciente y potente BAHA digital, tiene un sistema de cancelación de Feedback, éste es ideal para pacientes que necesitan más beneficios que los que brinda el BAHA DIVINO. Éste posee 3 programas de audición, procesamiento lineal, sistema de compresión con supresión de ruido y puede adaptarse al sistema FM, audio, etc. Batería 675, con señal de aviso cuando ésta necesita cambio. Ideal para pérdidas auditivas mixtas progresivas y pérdidas auditivas neurosensoriales unilaterales.

El BAHA Cordelle II: posee las mismas características del intenso, adicionalmente tiene conexión a telecoil, es un procesador de cuerpo, control de volumen con batería $9 \mathrm{~V}^{12}$.

A continuación se presentan las ventajas y desventajas de esta ayuda auditiva:

TABLA 1.

VENTAJAS Y DESVENTAJAS DEL BAHA.

\section{Ventajas}

No hay oclusión del oído externo permitiendo una buena ventilación de las cavidades de mastoidectomía.

No ofrece presión sobre la piel.

Buena aceptación a nivel mundial.

Aprovechamiento de la vía ósea como herramienta de rehabilitación auditiva.
Necesidad de una cirugía para su implante.

Tecnología en proceso de conocimiento mundial. Mayor costo comparativo con los audífonos convencionales.

Cuidados especiales alrededor del implante osteointegrado percutáneo.

Corto tiempo de garantía. omnidireccional.

Calidad de sonido significativamente superior.

Es fácil de limpiar.

Su ubicación es flexible.

Tratado de otología y audiología 2007, p. 659.

12 Cochlear. BAHA. A natural pathway to hearing. Americas and Canada.Dec. 2007. 


\section{Transmisión transcraneal}

Como el oído interno está en la porción petrosa del hueso temporal, las vibraciones de este hueso producirán movimientos en los líquidos del oído interno directamente. Por esta razón, la sensación auditiva puede ser producida en ausencia de vibraciones transmitidas a través de la membrana timpánica y la cadena osicular.

El mecanismo de transmisión del sonido por conducción ósea es menos efectivo que por vía aérea ya que la intensidad del sonido debe ser muchas veces mayor para poner en vibración el tejido óseo craneano; que para estimular el mecanismo de conducción a través de la membrana timpánica. Además la transmisión a través de la piel, los tejidos blandos y el hueso es menor para los sonidos de onda corta (alta frecuencia) que para los sonidos de onda larga (baja frecuencia). Por esta razón la audición por vía ósea tiende a ser algo distorsionada en comparación con la de la vía aérea.

Aunque finalmente cumple el mismo propósito, la deformación de la membrana basilar. Este fenómeno es lo que se llama la "conducción ósea inercial", para la frecuencia entre 800 y 1600 cps, entra en juego la elasticidad del cráneo de acuerdo con la ubicación de los puntos sonoros; mientras de un lado se produce un movimiento hacia delante, el lado opuesto a ese movimiento va hacia atrás $^{13}$.

Para evaluar esta vía, el vibrador óseo genera vibraciones del cráneo estimulando directamente la cóclea, por factores como la aceleración del hueso temporal debido a la inercia en la respuesta del mecanismo osicular y los fluidos del oído interno; las vibraciones distorsionadas del hueso temporal causadas por la conducción ósea por compresión y la energía irradiada en la parte ósea del Conducto Auditivo Externo (CAE). Las dife-

13 Rivas, Otología, Op. cit., pág. 123. rentes frecuencias viajan de manera distinta a través del tejido óseo hasta llegar a la cóclea.

Después de describir a grandes rasgos las ayudas audiométricas, es importante destacar algunos de los procedimientos de evaluación audiológica, los que son básicos en la elección de la prótesis auditiva.

\section{Enmascaramiento}

La audición cruzada se refiere a la característica por medio de la cual el sonido que estimula un oído, puede ser percibido en el oído contrario por vía aérea o por vía ósea. Para la conducción por vía ósea se ha determinado que con una diferencia de 5 a $10 \mathrm{~dB}$ en el umbral entre un oído y otro se puede presentar el mismo fenómeno. En este caso el sonido viaja a través del cráneo y estimula el oído contralateral. La manifestación del fenómeno de la audición cruzada, en el audiograma es lo que se ha llamado la "curva sombra" y es una gráfica casi superpuesta a la del otro oído, pero descendida varios $\mathrm{dB}$ y muestra cierta audición donde en realidad no existe ${ }^{14}$.

El enmascaramiento consiste en suministrar un ruido lo suficientemente fuerte por el oído contrario al evaluado o por el mismo oído, para obtener el umbral mínimo de audición real del oído evaluado, también se define como el hacer inaudible un sonido por la emisión de otro de mayor intensidad.

Hay dos condiciones que se deben respetar a la hora del enmascaramiento: El ensordecimiento debe enmascarar la audición coclear del oído a eliminar y no se debe enmascarar la audición coclear del oído interrogado (pues puede por sí mismo lateralizarse a partir de cierto nivel). La intensidad del ensordecimiento se sitúa entre dos valores siendo uno el mínimo de eficacia de ensordecimiento del oído a eliminar, y el otro al máximo de no repercusión en el oído a interrogar.

\footnotetext{
14 Rivas, Tratado de Otología y Audiología. Op cit., pág. 106.
} 
El sonido enmascarante debe ser eficaz para ensordecer al oído que se quiere eliminar: La intensidad mínima eficaz para ensordecer un oído debe disminuir el umbral óseo que esté por debajo del oído opuesto que no se evalúa, lo que se llama criterio de eficacia. Para esto, la intensidad del sonido enmascarante debe ser al menos igual al de ensordecimiento específico, más la intensidad del sonido con la cual se evalúa el otro oído.

Se sabe que un sonido introducido en el oído por vía aérea puede lateralizarse en el oído opuesto, si su energía es superior a $60 \mathrm{~dB}$, se le llama criterio de no repercusión. Así, el sonido enmascarante podrá repercutir sobre el oído opuesto si es superior a $60 \mathrm{~dB}$. La intensidad máxima no repercutora utilizable es, igual a la suma de la intensidad del sonido-test emitido en el oído interesado por conducción ósea y de la energía de transmisión transcraneana del sonido enmascarante emitido en conducción aérea; o sea, 60 dB.

\section{Oclusión}

Es una mejoría artificial en la respuesta de conducción ósea. Las mejores respuestas son el resultado de una presión sonora generada en el canal auditivo externo cerrado y transmitida a través del mecanismo conductivo. Esto está asociado con una energía adicional creada en la cóclea, la que se convierte, entonces, en un cambio verdadero en la sensibilidad. El efecto de oclusión ocurre cuando tapamos un oído normal o una pérdida sensorioneural ${ }^{15}$.

Cuando se presentan deficiencias auditivas, es importante realizar una adecuada evaluación para determinar la pertinencia de adaptar una ayuda auditiva, estimar cuanto se puede beneficiar el sujeto con ésta y determinar las características necesarias para una amplificación efectiva, lo que le va a permitir al candidato un máximo bienestar comunicativo ${ }^{16}$.

\footnotetext{
15 Gómez, Audiología básica. Op. cit., pág. 66.

16 Gómez Gómez, Olga. Audiología básica. Op cit., pág. 264.
}

En algunos casos este tipo de evaluaciones no se realizan adecuadamente, por lo cual se obtienen fracasos en cuanto a la ganancia de este tipo de ayudas auditivas, lo que hace que los usuarios no se sienten satisfechos ya que no les provee de los beneficios que esperaban obtener con estas ayudas.

En la actualidad no se conoce ningún protocolo de evaluación auditiva pre y posquirúrgica para candidatos a implante osteointegrado BAHA con hipoacusia neurosensorial profunda unilateral.

Entre las pocas investigaciones reportadas se encuentran las de Gómez y García (2006) realizada en Bogotá y la de Niparko, Wazen y Fayad (2002), realizada en la California, en éstas se describen la(s) técnica(s) que utilizan para evaluar el beneficio del implante BAHA. En la primera se utiliza la oclusión ${ }^{17}$ y en la segunda el enmascaramiento con la aplicación del protocolo de Satisfacción con la Amplificación en la Vida Diaria (Satisfaction whit Amplification in Daily Life) SADL $^{18}$.

A nivel mundial no existe hasta el momento un protocolo pre y posquirúrgico para este tipo de usuarios con hipoacusia neurosensorial profunda unilateral, encontrando el uso del enmascaramiento y la oclusión de CAE como los procedimientos más utilizados en la evaluación. Otro de los métodos con los cuales se evalúa a este tipo de población es exponiendo al usuario en diferentes contextos de escucha que permiten a éste de manera incidental medir su percepción ante el estí-

\footnotetext{
17 Gómez Vargas, Francy; García Plata, Eliana. Estudio comparativo del nivel de satisfacción del usuario de audífonos transcraneano y osteointegrado BAHA en pérdidas unilaterales neurosensoriales o mixtas en pacientes entre los 5 y 50 años de edad en Bogotá, 2006, Trabajo de grado (Especialista en Audiología). Fundación Universitaria Escuela Colombiana de Rehabilitación. Facultad de Fonoaudiología.

18 Niparko, John K.; Wazen, Jack; J. N. Sohan; Fayad, José N. Transcranial contralateral cochlear stimulation in unilateral deafness: Otolaryngology. Head and Neck Surgery .Vol. 129, $\mathrm{N}^{\circ} 3$.
} 
mulo sonoro del BAHA por medio de la aplicación del test de desempeño auditivo en ruido.

Anders Tjellstrom et al. (2008) hacen un test de desempeño, donde para la evaluación de esta población ellos le piden al paciente llevar el procesador BAHA con una soft band para usar durante una semana en las diferentes actividades de su vida diaria y al final con la cooperación del paciente en contestar determinados interrogantes, se evalúa la utilidad del dispositivo ${ }^{19}$.

Dentro de algunos de los programas consultados de evaluación a candidatos a Implante BAHA en Colombia, se encuentran la Clínica José A. Rivas, el Hospital Universitario San Ignacio, Clínica San Rafael, Clínica de Medellín, Instituto Cinda y la Fundación Santa Fe de Bogotá. Cada uno de estos centros aplica un protocolo de evaluación diferente y ha sido diseñado dependiendo de las características de sus pacientes.

La evaluación auditiva tiene un valor muy importante en el proceso diagnóstico de los pacientes, ya que por medio de este procedimiento se puede determinar el estado auditivo de una persona, por lo cual se debe realizar minuciosamente para determinar un posible tratamiento con el fin de obtener una mejor calidad en la percepción del sonido y para no crear falsas expectativas en la adaptación de las ayudas auditivas.

Teniendo en cuenta la información anterior se hace necesario indagar sobre los métodos de valoración más utilizados para realizar una comparación y determinar cuál de los dos es el más adecuado en la evaluación, ya que este proceso es muy importante para someter a un paciente a este tipo de procedimiento quirúrgico, el cual es invasivo ya que el BAHA es un dispositivo de titanio anclado al hueso del cráneo para el cual se requiere un pro-

19 Entrevista con Anders Tjellstrom, profesor del Departamento de Otorrinolaringología de la Universidad de Goteborg. Semana de la otología, Bogotá, abril de 2008. ceso de cirugía y también costoso ya que a parte del valor económico del implante, se ven otros factores como la ausencia laboral por la incapacidad de la cirugía y también va a generar en el usuario diferentes cambios a nivel social, cultural y psicológico ya que se va a enfrentar a un mundo donde las personas en algunas ocasiones pueden rechazar a un individuo por aspectos diferentes en la apariencia. Además este tipo de investigaciones aportan información y conocimientos sobre los diferentes procesos que se están utilizando en estos candidatos.

El número de personas con hipoacusia neurosensorial unilateral aumenta significativamente y suele pasar desapercibidas o adaptarse fácilmente a la pérdida de un oído ya que el escuchar por el otro hace que puedan desenvolverse social, laboral y escolarmente; sin embargo, esta dificultad, hace que el individuo presente problemas en situaciones de comunicación tales como: dificultad para entender sonidos suaves de lenguaje en ambientes de ruido, dificultad en la localización de sonidos ya que no pueden ubicar la dirección de donde provienen, dificultad para mantener la atención en clase y seguir instrucciones especialmente si hay ruido de fondo, dificultad con las tareas escolares, particularmente con los que emplean sonidos de lenguaje como la lectura y la escritura y dificultad para participar en actividades sociales como reuniones.

Según el Censo de Población 2005 DANE (Departamento Administrativo Nacional de Estadística), del total de población colombiana de 41'242.948 personas, 2’639.549 personas poseen algún tipo de discapacidad, ya sea física, sensorial o cognitiva, lo que representa el 6,4\% de la población. El dato específico de población en situación de discapacidad auditiva se ubica de manera complementaria a través de los registros correspondientes a las preguntas por "Limitaciones para oír aún con aparatos especiales", y "Limitaciones para hablar", a las que respondieron 456.642 personas y 337.862 personas, respectivamente. El resulta- 
do del cruce de estas dos últimas variables de quienes respondieron afirmativamente a las dos preguntas, arroja la identificación de 142.961 personas en situación de discapacidad auditiva.

La pérdida de la sensibilidad auditiva hoy en día es común ya que un gran número de la población mundial la padece, un estudio realizado por la ASHA (American Speech-Language-HearingAssociation) identificó que el número de norteamericanos con pérdida auditiva se ha duplicado durante los últimos 30 años. Los datos arrojados por investigaciones federales ilustran que: 13.2 millones de personas en 1971, 14.2 millones (1977), 20.3 millones (1991), y 24.2 millones (1993) y las últimas investigaciones estiman que 28.6 millones de norteamericanos tenían un desorden auditivo en $2000^{20}$.

En cuanto a la población infantil uno de cada 1.000 recién nacidos norteamericanos tienen pérdida auditiva unilateral UHL que no son detectados a tiempo sino hasta la edad escolar ${ }^{21}$.

Las personas con UHL, muestran que están en riesgo académico, social, emocional y requieren a menudo de servicios especiales para tratar estas necesidades. Aproximadamente de 16 a 19 de cada 1.000 niños en el colegio presentan $\mathrm{UHL}^{22}$, aunque es importante aclarar que las personas con pérdida auditiva bilateral tienen mayor compromiso a nivel social, académico y emocional que los individuos con UHL ya que su canal auditivo está deprivado en un porcentaje más alto.

Los sujetos con UHL no tienen la ventaja de la audición binaural y por lo tanto, tienen dificultad

20 American Speech - Hearing Association. The Prevalence and Incidence of Hearing Loss in Adults [online], 1997-2007. Asha.

21 Oyler, R. \& McKay, S. Unilateral hearing loss in children: Challenges and opportunities. The [online], 2005. Asha.

22 Brookhauser, Workthington y Nelly. 1991. Management Guidelines for children with unilateral hearing loss [online], 2005. para la localización de sonidos, discriminación y detección del discurso si éste es recibido por el oído que presenta la pérdida. De igual manera se les dificulta entender en ambientes ruidosos y reverberantes.

Resaltando la importancia de la evaluación para la adaptación de prótesis auditivas, dada la alta tasa de incidencia y prevalencia de hipoacusia, llama la atención que no existe, o no se conoce ningún protocolo de evaluación prequirúrgica ni posquirúrgica para determinar el beneficio que el BAHA pueda dar a los pacientes con hipoacusia neurosensorial profunda unilateral. Y además teniendo en cuenta que para el uso de esta prótesis auditiva, se hace mediante un proceso invasivo, que puede llevar riesgos, se destaca la necesidad de examinar la sensibilidad de las pruebas que con mayor frecuencia se utilizan.

Como se evidenció en el anterior párrafo al no existir un protocolo específico de evaluación para este tipo de población cada centro ha ideado la mejor técnica de evaluación para cada uno de los pacientes y es por esto que se crea la necesidad de realizar este tipo de investigación para encaminar un exitoso proceso de evaluación.

Y hasta el momento se desconoce un estudio que especifique un protocolo para la evaluación audiológica en estos candidatos, sólo se refieren a la ganancia y beneficio del BAHA en personas con determinada pérdida auditiva, es por esto que en el presente estudio, se compararán dos pruebas de uso generalizado, el enmascaramiento y la oclusión, lo que posteriormente ayudará a determinar el procedimiento más adecuado en la evaluación de pacientes candidatos al implante osteointegrado BAHA con hipoacusia neurosensorial profunda unilateral.

Es así como el objetivo de la presente investigación es comparar los umbrales auditivos de la técnica del enmascaramiento con la oclusión de los candidatos a implante BAHA con hipoacusia neurosensorial profunda unilateral. 


\section{Metodología}

\section{Tipo de investigación}

Esta investigación es de tipo comparativo ya que busca determinar los umbrales auditivos y contrastarlos con los procedimientos de enmascaramiento mínimo y máximo con la de oclusión en usuarios con hipoacusia neurosensorial profunda unilateral.

\section{Participantes}

Fueron 16 el total de la población con hipoacusia unilateral profunda de la Clínica José A. Rivas candidatos a BAHA, los cuales fueron evaluados otológicamente y se le descartó alguna patología retrococlear.

A continuación se presenta el género y la edad de los participantes.

Se encontró que el $25 \%$ de la población se incluía dentro de un rango de edad entre los 20 y 25 años y pertenecían al género femenino.

\section{Instrumentos}

- Otoscopio WelchAllyn.

- Audiómetro Amplaid 309 de dos canales con salida de campo libre.

- Material de impresión Precise II de Starkey para el procedimiento de oclusión.

- Inmitanciómetro Maico MI 34.

- Otoblocks.

- Otoluz.

\section{Procedimiento}

Se tomaron 30 personas que asisten a consulta audiológica en la Clínica José A. Rivas las cuales después de ser valoradas otológicamente fueron incluidas para la realización de esta investigación.

Inicialmente se realizó una invitación a las personas candidatas al estudio con hipoacusia neurosensorial profunda unilateral con conductos auditivos libre de cerumen y sin ninguna patolo-

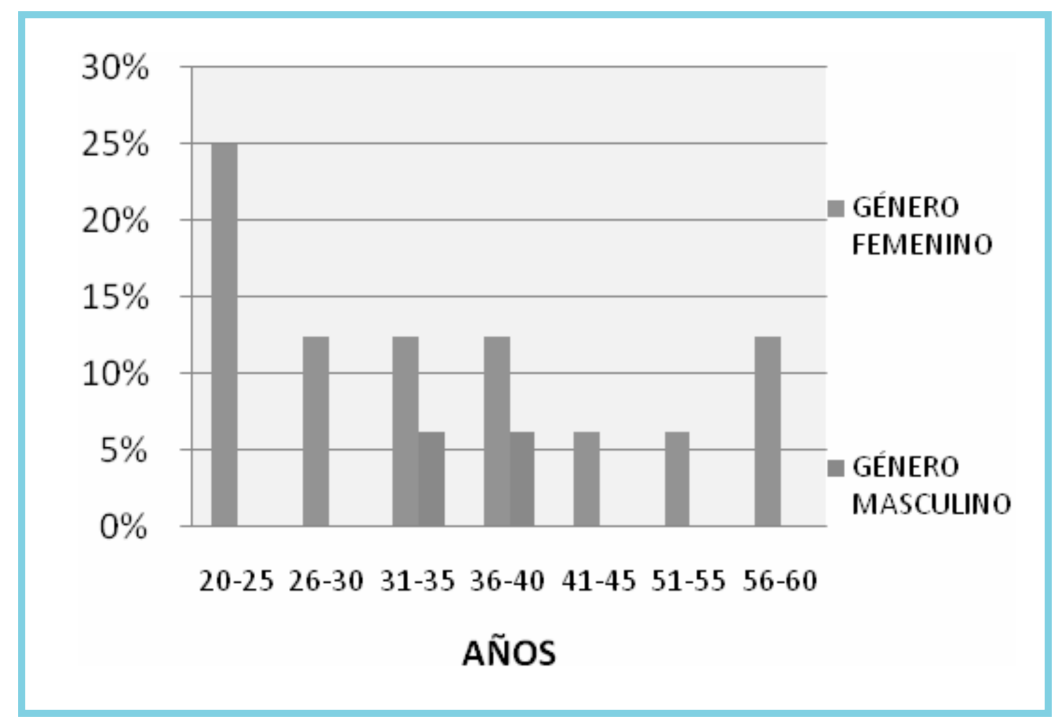

Figura 3. Edad - Género.

gía de oído medio, pero únicamente 16 de los 30 sujetos fueron evaluados ya que 2 de los pacientes fueron sometidos a procedimiento quirúrgico y la audición del oído hipoacúsico recuperó audición en un grado significativo, 8 de los pacientes no llegaron el día de la evaluación y finalmente a 4 se 
les dificultó asistir al procedimiento por razones laborales.

En los días que fueron citados los pacientes se le dio a cada uno, una orientación sobre el objeto del estudio, después decidían voluntariamente la participación en la investigación y firmaban el consentimiento informado. Según la resolución 8430 de 1993 del Ministerio de Salud en Reglamentación en Ciencia y Tecnología (1998). Esta investigación es de riesgo mínimo ${ }^{23}$.

Posteriormente se realizó otoscopia para evaluar el estado del oído externo (CAE) y membrana timpánica porque determinar algunas patologías del oído externo, luego la audiometría que evaluó los umbrales mínimos de audición de los candidatos al implante BAHA de la Clínica José A. Rivas, adicionalmente se realizó la logoaudiometría para establecer la discriminación del lenguaje, a continuación la inmitancia acústica para corroborar la función del oído medio.

Seguidamente se realizó la evaluación de audiometría tonal con el BAHA utilizando en el oído con la hipoacusia el procesador DIVINO en campo libre con el enmascaramiento; en donde se aplicó un ruido de $15 \mathrm{~dB}$ por encima del umbral en el oído con audición normal, teniendo en cuenta el criterio de eficacia y no repercusión y $30 \mathrm{~dB}$ por encima del umbral aéreo para el masking máxi$\mathrm{mo}^{24}$. Y finalmente el procedimiento de oclusión, el cual se realizó introduciendo material de impresión cubriendo totalmente el CAE. Para los dos procedimientos se tomaron los umbrales de las frecuencias $500 \mathrm{~Hz}, 1000 \mathrm{~Hz}, 2000 \mathrm{~Hz}$ y $4000 \mathrm{~Hz}$ con el parlante a 0 grados azimut.

\section{Resultados}

Los datos se analizaron en Excel con las pruebas de estadística descriptiva como media, desviación estándar, porcentajes y para la comparación de los umbrales se utilizó la prueba t Student.

Se tomaron los umbrales auditivos de las frecuencias de 500, 1000, 2000 y $4000 \mathrm{~Hz}$ con el BAHA procesador DIVINO en campo libre con el procedimiento de enmascaramiento y de la misma forma con el de oclusión.

A continuación se presentan los datos obtenidos en la audiometría bilateral, en la logoaudiometría, en el timpanograma, el tiempo y las causas de la pérdida auditiva

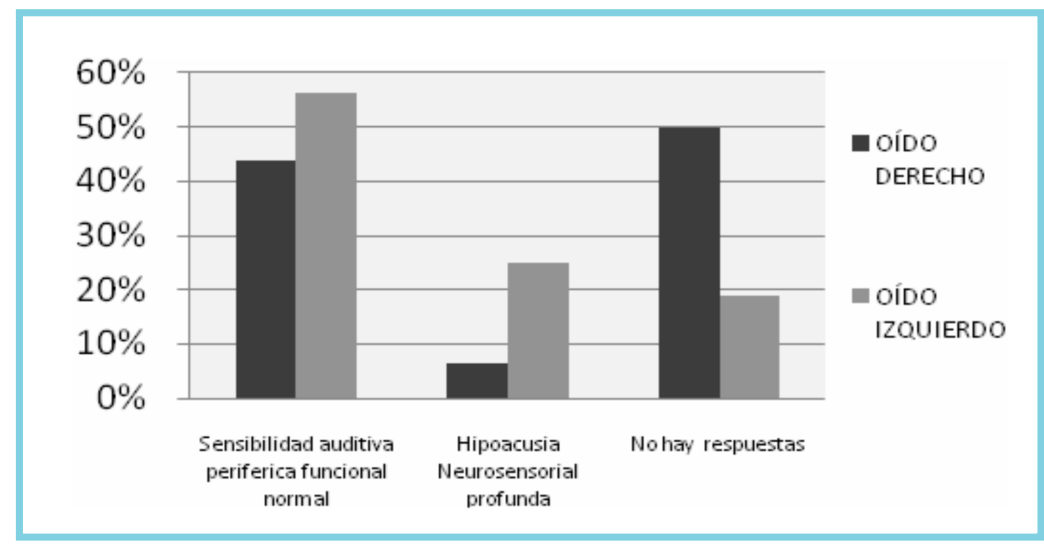

Figura 2. Interpretación de la audiometría bilateral.

23 Resolución 8430 de 1993 del Ministerio de Salud en Reglamentación en Ciencia y Tecnología. (1998). Ministerio de Salud, Dirección de Desarrollo Científico y Tecnológico, República de Colombia, Bogotá, D.C.

\footnotetext{
${ }^{4}$ Niparko, John K.; Wazen Jack J. PHD, N. Sohan; Fayad, José $\mathrm{N}$. Transcranial contralateral cochlear stimulation in unilateral deafness. Op. cit.
} 
Se encontró que el 43,70\% de la población tenía sensibilidad auditiva periférica funcional normal en el oído izquierdo y se encontró no respuesta en un $50 \%$ para el oído derecho.
Se encontró que la curva corrobora los hallazgos de la audiometría tonal en un $62,50 \%$ para oído izquierdo.

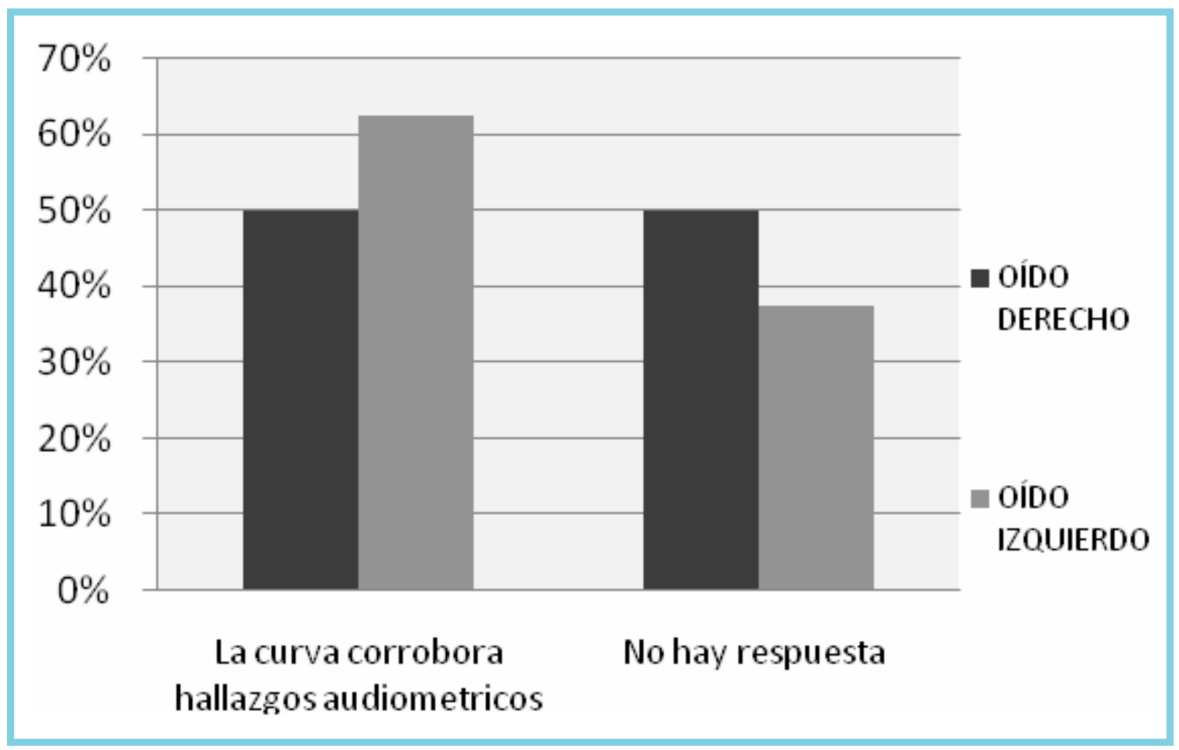

Figura 3. Interpretación de la logoaudiometría bilateral.

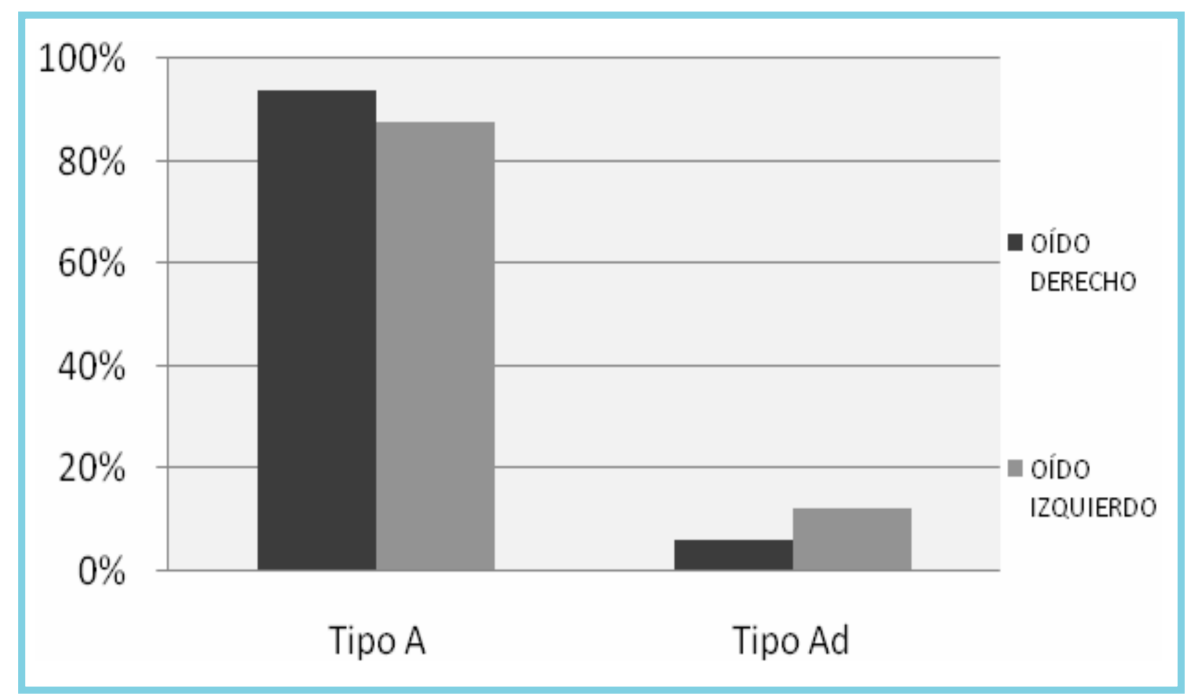

Figura 4. Interpretación timpanograma bilateral. 
Se encontró Tipo A funcional normal del oído medio para oído derecho en un 93,75\%.
Como se aprecia en la gráfica anterior, el $37,50 \%$ de la población tiene la pérdida auditiva hace más de 60 meses.

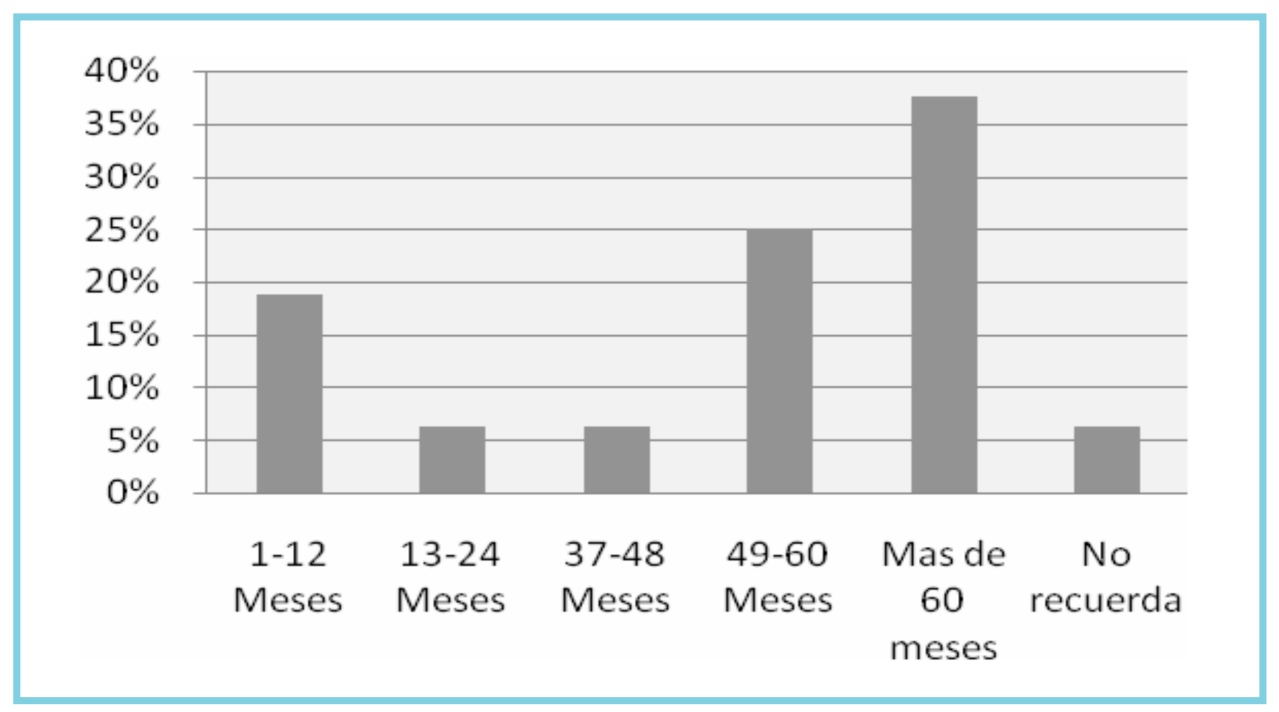

Figura 5. Tiempo de la pérdida auditiva.

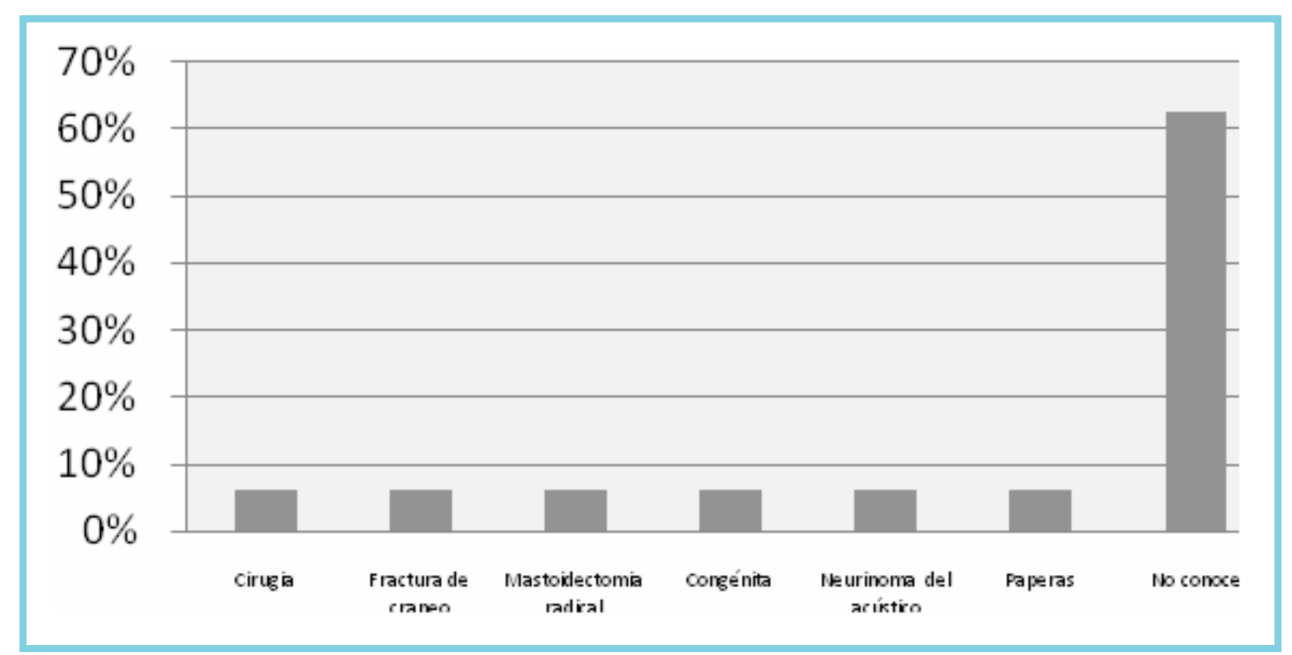

Figura 6. Causas de la pérdida auditiva.

El $62,50 \%$ de la población no conoce las causas de la pérdida auditiva, y una minoría de ellos, tiene diferentes causas que van en un amplio rango desde cirugías, fracturas del cráneo, hasta paperas.
A continuación se presentan los umbrales auditivos de la frecuencia de $500 \mathrm{~Hz} 1000 \mathrm{~Hz}, 2000$ $\mathrm{Hz}$ y $4000 \mathrm{~Hz}$ en campo libre con el método de enmascaramiento mínimo, máximo, oclusión, Masking mínimo vs. Oclusión y Masking máximo vs. Oclusión con el BAHA procesador DIVINO. 


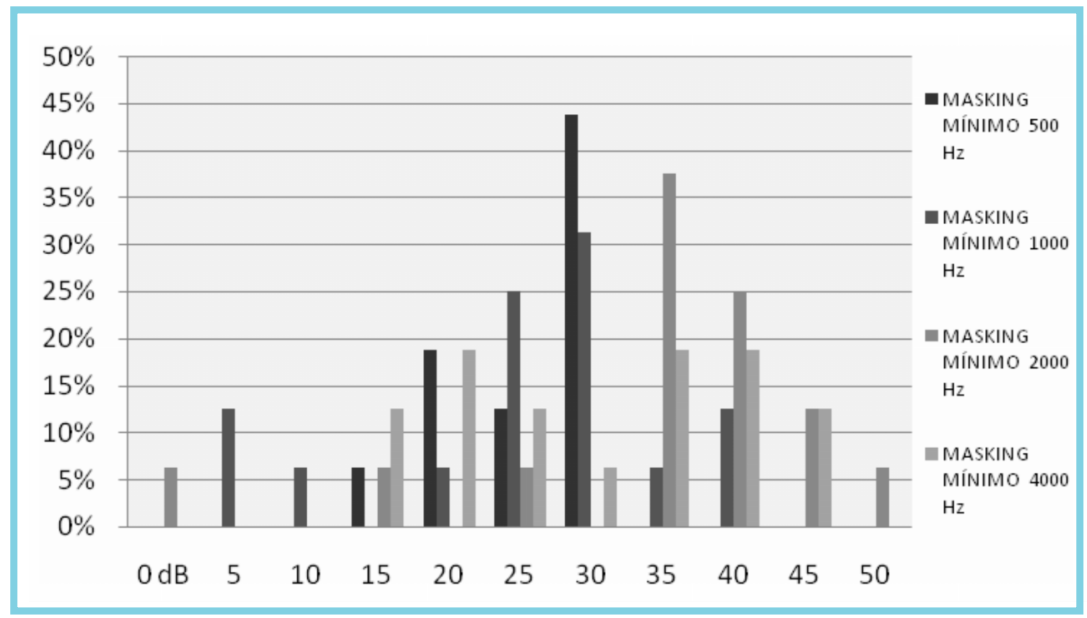

Figura 7. Masking mínimo.

Como se observa en la figura 9, en los sujetos evaluados con Masking mínimo se evidencian respuestas por encima y dentro del espectro del lenguaje para las frecuencias 500, 1000 y $2000 \mathrm{~Hz}$, para $4000 \mathrm{~Hz}$ se encontró el 12,50\% con respuestas fuera del espectro del lenguaje.
De acuerdo con los resultados obtenidos en los sujetos evaluados con Masking máximo se evidencian respuestas por encima y dentro del espectro del lenguaje para la frecuencia $500 \mathrm{~Hz}$ en todas las intensidades.

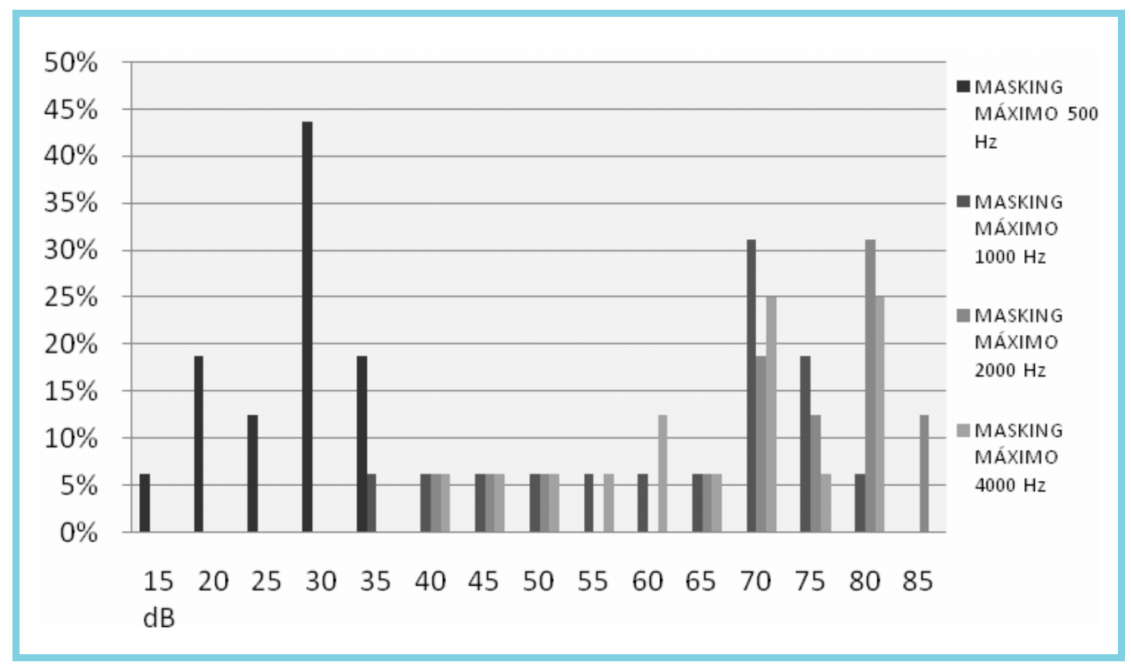

Figura 8. Masking máximo.

Para la frecuencia $1000 \mathrm{~Hz}$ se observan respuestas por encima y dentro del espectro del lenguaje para las intensidades de $35 \mathrm{a} 65 \mathrm{~dB}$, para las intensidades $70 \mathrm{~dB}(31,2 \%), 75 \mathrm{~dB}(18,7 \%)$ y 80 $\mathrm{dB}(6,2 \%)$ de las respuestas se encuentran fuera del espectro del lenguaje.
La frecuencia $2000 \mathrm{~Hz}$ se evidencian respuestas por encima y dentro del espectro del lenguaje para las intensidades de 40 a $50 \mathrm{~dB}$, las intensidades $65 \mathrm{~dB}(6,2 \%), 70 \mathrm{~dB}(18,5 \%), 75 \mathrm{~dB}(12,5 \%)$, $80 \mathrm{~dB}(31,2 \%)$ y $85 \mathrm{~dB}$ el $(12,5 \%)$ de las respuestas se encuentran fuera del espectro del lenguaje para esta frecuencia. 
Finalmente en la frecuencia $4000 \mathrm{~Hz}$ se alcanza para la intensidad $40 \mathrm{~dB}(6,2 \%)$ se logra una respuesta dentro del espectro del lenguaje, el resto de las respuestas se obtienen por fuera del espectro del lenguaje.
De acuerdo con los resultados obtenidos en los sujetos evaluados con oclusión se evidencian respuestas por encima y dentro del espectro del lenguaje para todas las frecuencias evaluadas.

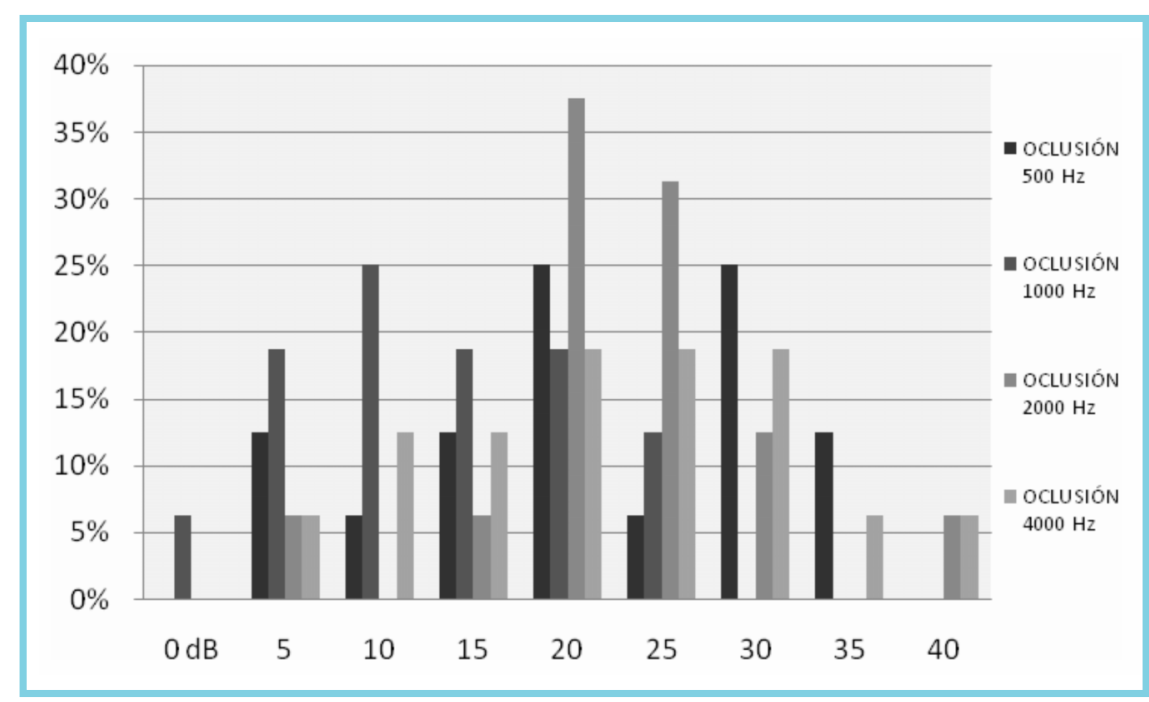

Figura 9. Umbrales auditivos mediante la técnica de la oclusión.

Seguidamente se presenta una tabla donde se resumen las comparaciones entre los dos procedimientos, el valor de t obtenido y la probabilidad asociada entre el Masking mínimo y la oclusión en las diferentes frecuencias establecidas.

Tabla 2.

Comparación entre Masking mínimo vs. OClusión, SE PRESENTA LA T Y LA PROBABILIDAD ASOCIADA

\begin{tabular}{c|c|c|c|c}
\hline Frecuencias & Masking mínimo & Oclusión & t de Student & P \\
\hline $500 \mathrm{~Hz}$ & $27.50 \pm 6.05$ & $21.56 \pm 9.78$ & 2,00 & 0,06 \\
\hline $1000 \mathrm{~Hz}$ & $25.31 \pm 10.71$ & $13.12 \pm 7.50$ & 4,87 & $0,00 *$ \\
\hline $2000 \mathrm{~Hz}$ & $34.37 \pm 12.23$ & $22.81 \pm 7,52$ & 2,99 & $0,00 *$ \\
\hline $4000 \mathrm{~Hz}$ & $30.31 \pm 10.40$ & $22.18 \pm 9.65$ & 2,40 & $0,02 *$ \\
\hline
\end{tabular}

* Significativamente menor que $\mathrm{p}=0.05$.

Para el procedimiento Masking mínimo comparado con el procedimiento de oclusión en las frecuencias $1000 \mathrm{~Hz}, 2000 \mathrm{~Hz}$ y $4000 \mathrm{~Hz}$, se encontraron diferencias significativas $\mathrm{t}=4.87 ; \mathrm{P}<$
$0.000 ; \mathrm{t}=2.99 ; \mathrm{P}<0.000$ y t= 2.4; $\mathrm{P}<0.02$, respectivamente encontrándose la oclusión con las mejores respuestas a los umbrales aéreos. 


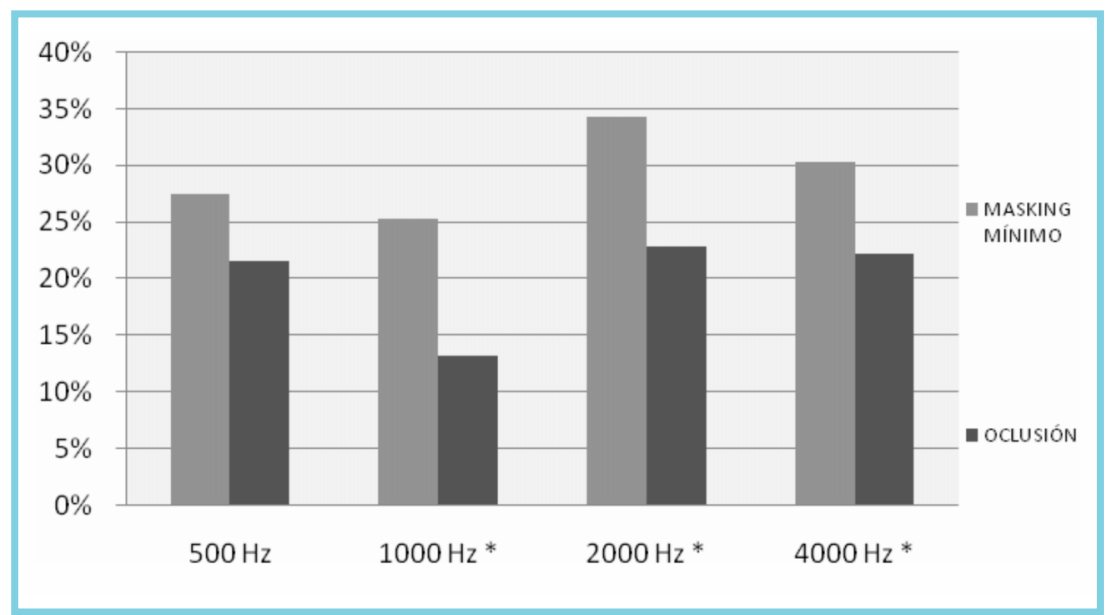

Figura 10. Masking mínimo vs. Oclusión.

En la única frecuencia en la cual no se encontraron diferencias entre estos dos procedimientos fue en la de $500 \mathrm{~Hz}$, en las demás la oclusión permitió obtener los mejores umbrales aeréos.

TABla 3.

Comparación entre Masking máximo vs. OClusión. SE PRESENTA LA T Y LA PROBABILIDAD ASOCIADA

\begin{tabular}{c|c|c|c|c}
\hline Frecuencias & Masking máximo & Oclusión & t de Student & P \\
\hline $500 \mathrm{~Hz}$ & $62.81 \pm 7.95$ & $21.56 \pm 9.78$ & 7.55 & $0.000^{* *}$ \\
\hline $1000 \mathrm{~Hz}$ & $62.81 \pm 13.78$ & $13.12 \pm 7.50$ & 8.58 & $0.000^{* *}$ \\
\hline $2000 \mathrm{~Hz}$ & $70.62 \pm 14.00$ & $22.81 \pm 7.52$ & 12.09 & $0.000^{* *}$ \\
\hline $4000 \mathrm{~Hz}$ & $65.62 \pm 12.89$ & $22.18 \pm 9.65$ & 2.40 & $0.02^{*}$ \\
\hline
\end{tabular}

* Significativamente menor que p 0.05 .

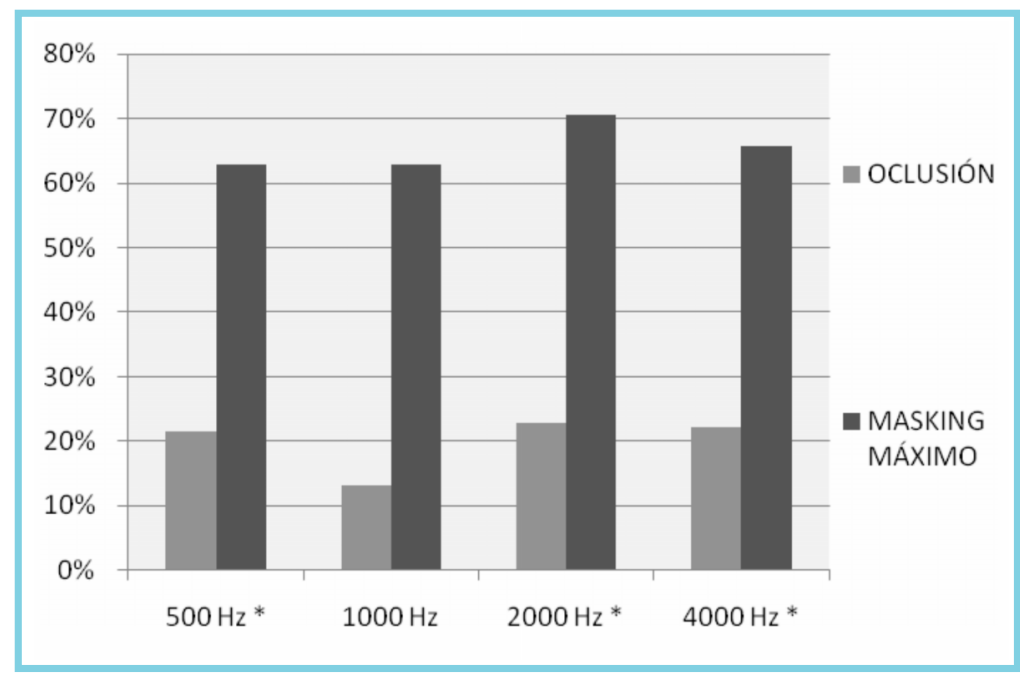

Figura 13. Masking máximo vs. Oclusión. 
Al realizar la comparación entre el método de Masking máximo y el de oclusión se evidencian diferencias significativas en todas las frecuencias evaluadas, así para la de $500 \mathrm{~Hz}, \mathrm{t}=7.55 ; \mathrm{p}=0.000$; para la de $1000 \mathrm{~Hz} \mathrm{t}=8.58$; $\mathrm{p}=0.000$; para la de $2000 \mathrm{~Hz}$ t=12.09; $\mathrm{p}=0.000$ y para la de $4000 \mathrm{~Hz}$, $\mathrm{t}=2,4 ; \mathrm{p}=0.05$; mostrando el procedimiento de oclusión ser el mejor con respuestas a los umbrales aéreos.

\section{Conclusiones}

En la comparación de estos procedimientos, el de enmascaramiento con el de la oclusión en la evaluación audiológica de candidatos a implante BAHA con hipoacusia neurosensorial profunda unilateral; se encuentra que la oclusión es el mejor procedimiento porque los umbrales obtenidos, fueron más bajos por lo que se ve este procedimiento sería el recomendable para la evaluación.

Todas las frecuencias evaluadas, en las cuales el procedimiento de oclusión muestra superioridad respecto del enmascaramiento, se encuentran dentro del espectro del lenguaje, de $500 \mathrm{a} 4000 \mathrm{~Hz}$. $\mathrm{Y}$ es, el procedimiento de oclusión el que hace que se obtengan mejores respuestas a los umbrales aéreos para todas las frecuencias evaluadas.

Al realizar la evaluación de los umbrales aéreos con el procedimiento de enmascaramiento mínimo, se obtienen respuestas dentro del espectro del lenguaje para las frecuencias $500 \mathrm{~Hz}, 1000$ $\mathrm{Hz}$ y $2000 \mathrm{~Hz}$, para las frecuencias $4000 \mathrm{~Hz}$ se obtienen respuestas por fuera del espectro del lenguaje, ya que el enmascaramiento atenúa afectando el umbral de la cóclea que está respondiendo y a partir de la cual se está aplicando el efecto de transmisión transcraneal al oído con hipoacusia profunda.

Lo mismo se encuentra con el enmascaramiento máximo.

Al analizar los resultados obtenidos con el procedimiento de oclusión en donde se evidenciaron respuestas dentro del espectro del lenguaje para todas las frecuencias, se evidencia que en este procedimiento se afecta el umbral aéreo del oído sano pero se mantiene el umbral óseo de éste beneficiando así la transmisión transcraneal.

La evaluación en cabina de los umbrales aéreos con el procesador del BAHA es un procedimiento importante ya que por medio de éste se estima el beneficio auditivo que puede brindar el BAHA al paciente antes de la cirugía.

Al aumentar el sonido enmascarante en la evaluación se puede evidenciar que la transmisión transcraneal tiende a disminuir, por lo tanto, se obtienen menores respuestas en los umbrales aéreos.

Al realizar un mal procedimiento de evaluación se pueden dar falsas expectativas al paciente sobre el beneficio real que puede ofrecer el BAHA. Ya que ese es el momento donde se identifica la ganancia que puede dar el BAHA en este tipo de hipoacusia.

No fue posible llevar a cabo este estudio con una muestra amplia de pacientes con hipoacusia neurosensorial profunda unilateral, debido a diferentes factores como el predominio de patología bilateral, sujetos con cirugías realizadas recientemente, muchos de los pacientes no fueron ubicados fácilmente, lo cual fue una limitante para tener contacto con ellos.

Teniendo en cuenta que ya se realizó el estudio para determinar cuál es el método más efectivo para hallar los umbrales aéreos en cabina con el BAHA, sería indicado realizar un estudio con el método de oclusión y la aplicación del protocolo de Satisfacción con la Amplificación en la Vida Diaria (Satisfaction whit Amplification in Daily Life) SADL, con pacientes ya implantados.

Recibido: agosto 2008

Aceptado: octubre 2008 


\section{Bibliografía}

ASHA, American Speech - Hearing Association [online] The Prevalence and Incidence of Hearing Loss in Adults [online], 1997-2007.

Arlinger, S. Negative consequences of uncorrected hearing loss - a review. University Hospital, Department of Audiology [online], July 2003. Pub med - indexed for Medline Asoaudio, audiología en general [online]. www.asoaudio.org.co.

Brookhauser, Workthington y Nelly. 1991. Management Guidelines for children with unilateral hearing loss [online], 2005.

Carcedo Gracia, Luis María. Otología. Buenos Aires Madrid: Panamericana, 2004; 80, 280292-319.

Cochlear, Corporation, Audiologist Manual Selection criteria, [online]. Evaluation and fitting procedures for BAHA. 2003.

Cochlear. BAHA. A natural pathway to hearing. [online]. Americas and Canada. Dec. 2007. www.cochlear.com.

Cochlear, Cómo funciona el BAHA [online]. ww.spain.cochlear.com/Products/1755.asp - 8k
Tjellstrom, Anders. Comunicación personal. Semana de la otología, Bogotá, abril de 2008.

Gómez Gómez, Olga. Audiología básica. Universidad Nacional de Colombia, Bogotá, Colombia: 2006; 66, 72, 73, 264 y 214.

Hernadez Sampieri, Roberto. Metodología de la investigación. México. Editorial McGrawHill. 1998.

Niparko, John; K. Wazen; Jack J. PHD, N. Sohan; Fayad, José N. Transcranial contralateral cochlear stimulation in unilateral deafness: Otolaryngology-Head and Neck Surgery. Vol. 129, $\mathrm{N}^{\mathrm{o}} 3$.

Oyler, R. \& McKay, S. Unilateral hearing loss in children: Challenges and opportunities. The [online], 2005.

Resolución 8430 de 1993 del Ministerio de Salud en Reglamentación en Ciencia y Tecnología. (1998). Ministerio de Salud, Dirección de Desarrollo Científico y Tecnológico, República de Colombia, Bogotá, D.C.

Rivas, José A. Tratado de Otología y Audiología. Bogotá, Colombia: Amolca, 2007; 9, 94, 106, 123, 618 y 620. 